\title{
Identification and study of InV as an inverse autotransporter family representative in Edwardsiella piscicida
}

\author{
Yu Han ${ }^{1} \cdot$ Lifan Wei $^{1} \cdot$ Jingfan Xiao ${ }^{1} \cdot$ Yuanxing Zhang ${ }^{1,2,3} \cdot$ Qiyao Wang $^{1,2,3} \cdot$ Mian Zhou $^{1}$ (I)
}

Received: 25 February 2019 / Revised: 17 December 2019 / Accepted: 26 December 2019 / Published online: 12 February 2020

(c) Springer-Verlag GmbH Germany, part of Springer Nature 2020

\begin{abstract}
Invasins and intimins, members of virulence-related adhesin family which is involved in attachment and adherence to epithelial cells during infection, are found in various pathogens. These pathogens can attach to enterocytes and lead to the formation of a pedestal-like structure. Invasins and intimins belong to type Ve secretion systems, and the N-terminal $\beta$-barrel domain acts as a translocation pore to secrete the C-terminal passenger domain. However, the relationship between invasins/ intimins and type III secretion system (T3SS) has been poorly studied. Based on the transposon insertion mutant library of Edwardsiella piscicida, we got a transposon insertion mutant with significant T3SS defect and identified the mutated gene ETAE_0323 (named inV later). This gene encoded a protein with 2359 amino acid residues and was predicted to be an invasin. To study the relationship between InV and T3SS, strains with $\mathrm{N}$-terminus or C-terminus deleted InV fragments were made. However, none of them was able to copy the phenotype of the transposon insertion mutant previously identified. The localization of InV in $\Delta \mathrm{T} 3 \mathrm{SS}$ strain was not significantly different from WT, suggesting that the T3SS defect in the transposon insertion mutant was likely to be caused by polar effect. Nevertheless, depletion of in $V$ still showed dramatic internalization and virulence defect in HeLa cell and zebrafish model, respectively, suggesting $\mathrm{InV}$ as a virulence related protein.
\end{abstract}

Keywords InV $\cdot$ Edwardsiella piscicida $\cdot \mathrm{T} 3 \mathrm{SS} \cdot$ Invasin $\cdot$ Virulence

\section{Introduction}

Virulence factors are factors or agents released by a pathogen to infect the host and maintain the disease state once an infection has been established. Virulence factors are essential for the invading bacteria to colonize, invade host cells, adapt to various environments in the host, subvert host

Communicated by Erko Stackebrandt.

Qiyao Wang

oawqiyao@ecust.edu.cn

$\triangle$ Mian Zhou

mianzhou@ecust.edu.cn

1 State Key Laboratory of Bioreactor Engineering, East China University of Science and Technology, 130 Meilong Road, Shanghai 200237, China

2 Shanghai Collaborative Innovation Center for Biomanufacturing, 130 Meilong Road, Shanghai 200237, China

3 Shanghai Engineering Research Center of Maricultured Animal Vaccines, Shanghai, China functions, and overcome the defenses of the host (Martens and Demain 2017). Invasins and intimins are members of virulence factors secreted by gram-negative pathogens. Currently, many invasins and intimins have been identified in pathogens including Yersinia pseudotuberculosis, Yersinia pestis, Escherichia coli, Salmonella enterica, Hafnia alvei, etc. (Leo et al. 2015; Chauhan et al. 2016), which played a crucial role in their attachment and invasion to intestinal epithelial cells (Oberhettinger et al. 2012; Tsai et al. 2010). These pathogens can intimately adhere to the colonic epithelium of the host and form a pedestal-like structure, disrupting the microvilli on the enterocyte surface. Intimins bind with its receptor Tir with high affinity, resulting in actin reorganization in the host cell (Leo et al. 2012; Gillenius and Urban 2015; Heinz et al. 2016). Tir is synthesized by the pathogen itself, and injected into the host cell via T3SS in advance. Invasins, however, directly bind to the host receptor $\beta 1$ integrins, triggering the re-arrangement of the host cell cytoskeleton to help with pathogen invasion (Leo et al. 2012; Isberg and van Nhieue 1995).

Invasins and intimins share common domains and can facilitate the invasion of pathogens into host cells (Fairman 
et al. 2012). Both invasins and intimins are mainly composed of four parts: signal peptide, periplasmic domain, translocation domain, and passenger domain (Leo et al. 2015). Signal peptide (SP), located at the N-terminus of the protein, mediates its transport and secretion through the Sec secretion system (Leo et al. 2015). Periplasmic domain, referred as the $\alpha$-domain, links the $\beta$-barrel domain. In addition, the lysin motif (LysM) in the periplasmic domain could bind to peptidoglycan under low pH conditions (Buist et al. 2010). The $\beta$-barrel and the linker region constitute the translocation domain, which is the transmembrane region and can stabilize the membrane due to $\alpha$-helical structures (Sadana et al. 2018). The passenger domain of invasins/intimins consists of a series of immunoglobulin (Ig)-like domains (Batchelor et al. 2000).

Invasins/intimins have been classified as inverse autotransporter proteins (IATs) due to the similar characteristics with autotransporter proteins (ATs). ATs have $\mathrm{N}$-terminal passenger domain and $\mathrm{C}$-terminal $\beta$-barrel domain, while IATs are opposite (Leibiger et al. 2019; Van Ulsen et al. 2018). Type V secretion system is the most common secretion system of gram-negative pathogens, which could be divided into five subtypes ( Va-Ve). Type Va systems are classical ATs; type Vb systems are two-partner secretion systems since the passenger domain and the translocation domain are two separate protein chains but are expressed from the same operon structure; type Vc systems are trimeric ATs; type Vd systems are recently described and similar to type Va systems; type Ve systems are IATs. Besides the inversion of passenger and $\beta$-barrel domains, type Ve systems also have a small peripheral domain at the N-terminus of the polypeptide chain. In some cases it contains a peptidoglycan-binding motif (Fan et al. 2016).

Edwardsiella piscicida, phylogenetically close to other model enteric pathogens such as pathogenic E. coli, Shigella and Salmonella species (Yang et al. 2012), is a bacterial pathogen with broad host (Xiao et al. 2008). E. piscicida mainly affects freshwater and marine animals, causing edwardsiellosis in over 20 fish species and resulting in large economic losses in the aquaculture industry worldwide (Leotta et al. 2009; Green 2010; Park et al. 2012; Leung et al. 2019). In our previous study, sequencing of the complete genome of a typical highly virulent $E$. piscicida strain EIB202 was accomplished (Wang et al. 2009). The genomics study revealed that $E$. piscicida harbored both type III and type VI secretion systems (T3SS and T6SS) (Tan et al. 2002; Srinivasa Rao et al. 2003; Zheng et al. 2005; Leung et al. 2012; Gao et al. 2015; Okuda et al. 2009). In E. piscicida, T3SS and T6SS are identified as two of the most important virulence mechanisms which are essential for bacterial internalization, survival and replication inside host cells (Srinivasa Rao et al. 2004). T3SS mutation or T6SS mutation which abolishes the secretion ability results in severely impaired cell invasion capabilities (Yang et al. 2015). The gene cluster of the T3SS in E. piscicida includes 34 genes, mainly encoding translocon proteins (EseB, EseC, and EseD), chaperones (EscA, EscB and EscC), effectors (EseG, EseH, EseJ, EseK), regulators (EsrA, EsrB and EsrC) and apparatus (EsaB, EsaN). T3SS forms syringe-like structure, and relies on translocon proteins to translocate effectors into the host cell (Wei et al. 2018). It has been reported recently that the balanced role of T3SS and T6SS are required to achieve full virulence ( $\mathrm{Hu}$ et al. 2019).

A transposon insertion mutant library based on Himarl was constructed to explore the upstream mediators of T3SS. Himarl transposon, recognizing TA sites, belongs to the mariner family and was initially isolated from the horn fly Haematobia irritans (Picardeau 2010). Luckily, ETAE_0323, annotated as an invasin, was screened out and showed critical activation towards T3SS secretion and expression. Here, we comprehensively investigated the function of ETAE_0323 and found that the mutant of ETAE_0323 showed attenuated internalization in HeLa cells and virulence defect in zebrafish model.

\section{Materials and methods}

\section{Bacterial strains and culture conditions}

The bacterial strains and plasmids used in this study are listed in Table 1. E. coli and E. piscicida were cultured in Luria-Bertani (LB) broth at $37^{\circ} \mathrm{C}$ and in LB or Dulbecco's modified Eagle's medium (DMEM) at $30^{\circ} \mathrm{C}$, respectively. When required, antibiotics were added at the following final concentrations: gentamicin ( $\mathrm{Gm} ; 15 \mu \mathrm{g} / \mathrm{mL})$, polymyxin $\mathrm{B}$ (Col; $20 \mu \mathrm{g} / \mathrm{mL}$ ), ampicillin (Amp; $50 \mu \mathrm{g} / \mathrm{mL}$ ), kanamycin (Kan; $50 \mu \mathrm{g} / \mathrm{mL}$ ), and chloramphenicol (Cm; $25 \mu \mathrm{g} / \mathrm{mL}$ ).

\section{Construction of inV in-frame deletion mutants and complementation strains}

All primers used in this study are listed in Table 2. As for in $V$ in-frame deletion mutants, we constructed both EIB202- $\Delta i n V-N^{\prime}$ and EIB202- $\Delta i n V-C^{\prime} . \Delta i n V-N^{\prime}$ had LysM and $\beta$-barrel domains deleted (1-452 aa), while $\Delta i n V-C^{\prime}$ had Big_3_5 domains deleted (692-2359 aa). PCR was performed to amplify upstream (primers sets 0323-C'-P1/0323-C'-P2 for $\Delta i n V-C^{\prime}, 0323-N^{\prime}-\mathrm{P} 1 / 0323-$ $\mathrm{N}^{\prime}$-P2 for EIB202- $\left.\Delta i n V-N^{\prime}\right)$ and downstream sequences (primer sets 0323-C'-P3/0323-C'-P4 for $\Delta i n V-C^{\prime}$, 0323-N'-P3/0323-N'-P4 for EIB202- $\Delta i n V-N^{\prime}$ ) of the deleted domains, and fusion PCR was used to combine these two fragments. Products were then cloned into the suicide vector pDM4 at SacI and SaII sites. The recombinant plasmids were amplified by $E$. coli $\mathrm{DH} 5 \alpha$ and then 
Table 1 Strains and plasmids used in this study

\begin{tabular}{|c|c|c|}
\hline Strains or plasmids & Genotype & References \\
\hline \multicolumn{3}{|c|}{ Edwardsiella piscicida } \\
\hline EIB202 $\Delta \mathrm{P}$ & EIB202, pEIB202 cured, used as wild type, $\mathrm{Col}^{\mathrm{r}}$ & Leibiger et al. (2019) \\
\hline$\Delta \mathrm{T} 3 \mathrm{SS}$ & EIB202, in-frame deletion of eseBCD, $\mathrm{Col}^{\mathrm{r}}$ & Tan et al. (2002) \\
\hline$\Delta e s r B$ & EIB202, in-frame deletion of esrB, $\mathrm{Col}^{\mathrm{r}}$ & Tan et al. (2002) \\
\hline in $V:: \operatorname{Tn}$ & EIB202, insertion disrupt by a MKGR transposon of inV, $\mathrm{Col}^{\mathrm{r}}$ & Lab collection \\
\hline$\Delta \operatorname{in} V-N^{\prime}$ & This study & \\
\hline$\Delta i n V-C^{\prime}$ & This study & \\
\hline$\Delta \operatorname{in} V-N^{\prime+}$ & This study & \\
\hline WT-inV- $N^{+}$ & This study & \\
\hline$\Delta \mathrm{T} 3 \mathrm{SS}-\mathrm{in} V-N^{+}$ & This study & \\
\hline \multicolumn{3}{|l|}{ Escherichia coli } \\
\hline DH5 $\alpha$ ipir & Host for $\pi$ requiring plasmids & Tan et al. (2002) \\
\hline SM10 $\lambda$ pir & Host for $\pi$ requiring plasmids, conjugal donor & Tan et al. (2002) \\
\hline \multicolumn{3}{|l|}{ Plasmids } \\
\hline pDM4 & Suicide plasmid, pir dependent, $\mathrm{R} 6 \mathrm{~K}, \mathrm{SacBR}, \mathrm{Cm}^{\mathrm{r}}$ & Tsai et al. (2010) \\
\hline pAKgfp1 & pBBRMCS4 with gfpmut3a, Amp ${ }^{r}$ & Srinivasa Rao et al. (2004) \\
\hline
\end{tabular}

transformed into $E$. coli SM10 $\lambda$ pir for conjugation with EIB202. Positive transconjugants were selected on LB plates containing $\mathrm{Cm}$ and Col. To complete the allelic exchange for in-frame deletion, double-crossover events were selected under $12 \%$ sucrose. Finally the deletion mutants were confirmed by PCR and sequencing.

To construct in $V$ complementation strains, the N-terminus in $V$ gene (1-452 aa) fused with flag tag was amplified (primer sets PAK-0323-F/PAK-0323-R) and cloned into pAK vector at KpnI and HindIII sites. The recombinant plasmid was amplified by $E$. coli DH5 $\alpha$ and then transformed into $E$. coli SM10 $\lambda$ pir for conjugation with wild-type EIB202, EIB202- $\Delta$ T3SS and EIB202- $\Delta i n V-N^{\prime}$ strains, respectively. Successfully complementation strains were screened on LB agar medium containing Amp and Col. Finally the complementation strains were confirmed by PCR and sequencing. The strains are named WT-inV$N^{\prime+}, \Delta \mathrm{T} 3 \mathrm{SS}-i n V-N^{+}$and $i n V-N^{+}$.

\section{Autoaggregation assays}

The autoaggregation assays were performed as previously described (Gao et al. 2015). Briefly, bacterial cells were cultured in $\mathrm{LB}$ medium at $30{ }^{\circ} \mathrm{C}$ up to $1.0 \mathrm{OD}_{600}$, and then diluted at a 1:100 ratio into fresh DMEM and incubated for $24 \mathrm{~h}$ at $30{ }^{\circ} \mathrm{C}$ without shaking. After that aggregation of phenotypes was recorded by a camera (D5600; Nikon, Japan). The autoaggregation assays were performed either in 96-well plates for large scale screening, or in glass test tubes for phenotype confirmation.

\section{Quantitative real-time PCR (qRT-PCR)}

The qRT-PCR assay was performed as previously described (Liu et al. 2018). All primers used in qRT-PCR are listed in Table 2. Briefly, bacterial cells were cultured in DMEM at $30{ }^{\circ} \mathrm{C}$ for $12 \mathrm{~h}$ without shaking, and then RNA was extracted using Simply P Total RNA Extraction Kit (BioFlux). cDNA was synthesized using FastKing RT Kit (TIANGEN). The qRT-PCR was performed on the 7500 Real-Time PCR system. The comparative $C_{\mathrm{T}}\left(2^{-\Delta \Delta C_{\mathrm{T}}}\right)$ method was used to quantitatively determine the transcriptional levels with the housekeeping $g y r B$ gene as an internal standard in each strain.

\section{Western blot analysis}

Sodium dodecyl sulfate polyacrylamide gel electrophoresis (SDS-PAGE) was performed to separate proteins, which then were transferred onto PVDF membrane (Millipore). The membranes were blocked for $2 \mathrm{~h}$ with $5 \%$ milk powder dissolved in the PBST buffer solution and probed with antiEseB (GL Biochem), anti-Dnak (Santa Cruz Biotechnology), anti-Flag rabbit antibody (Santa Cruz Biotechnology) and a peroxidase-conjugated secondary anti-rabbit (Beyotime) antibody.

\section{Preparation for protein and cytoplasm samples}

Bacterial cells were cultured in $\mathrm{LB}$ medium at $30{ }^{\circ} \mathrm{C}$ for $12 \mathrm{~h}$, then bacterial cells were added to protease inhibitor (PMSF) in advance, and were centrifuged to collect cells and cell-free supernatants. Whole cellular protein (WCP) 
Table 2 Primers used in this study

\begin{tabular}{|c|c|}
\hline Primers & Sequence $\left(5^{\prime}-3^{\prime}\right)$ \\
\hline $0323-\mathrm{C}^{\prime}-\mathrm{P} 1$ & CCCCCCCGAGCTCAGGTTACCCGGATCTATTTTATCCCTGCCCAGCGACAC \\
\hline 0323-C'-P2 & GCCTGTTTTACCGTTGGCGACGATGCTCTT \\
\hline $0323-\mathrm{C}^{\prime}-\mathrm{P} 3$ & TCGCCAACGGTAAAACAGGCTATACATTAAAG \\
\hline 0323-C'-P4 & GAGTACGCGTCACTAGTGGGGCCCTTCTAGGGCGACAGTGGTACGGCGTAC \\
\hline $0323-C^{\prime}$-in-F & GGCGTTCACATCAGCAATACCA \\
\hline 0323-C'-in-R & GATGCTAACACCGTTCGTTTCG \\
\hline $0323-C^{\prime}$-out-F & TAGCGAGCATGATACCCAATT \\
\hline $0323-\mathrm{C}^{\prime}$-out-R & CCTTCCTGTATTCTCCGTGAT \\
\hline $0323-\mathrm{N}^{\prime}-\mathrm{P} 1$ & GTGGAATTCCCGGGAGAGCTCGCCCTCAGTTAGCCGTAT \\
\hline $0323-\mathrm{N}^{\prime}-\mathrm{P} 2$ & CCGCGATTTCCATACTCGTTTACACCTGTTC \\
\hline $0323-\mathrm{N}^{\prime}-\mathrm{P} 3$ & AACGAGTATGGAAATCGCGGCCAATACTTA \\
\hline $0323-\mathrm{N}^{\prime}-\mathrm{P} 4$ & AAGCTTATCGATACCGTCGAGTTGGCGACGATGCTCTT \\
\hline $0323-\mathrm{N}^{\prime}$-in-F & ACGCACAATCAACTGAACCC \\
\hline 0323-N'-in-R & AAATAGCCCTACCGCATCCC \\
\hline $0323-\mathrm{N}^{\prime}$-out-F & TTTTATCGTCTCGGTATCCAAC \\
\hline $0323-\mathrm{N}^{\prime}$-out-R & CTGCTAATGAACGCCACCTT \\
\hline pDM4-F & AACAAGCCAGGGATGTAACGC \\
\hline pDM4-R & TCCAGTGGCTTCTGTTTCTA \\
\hline PAK-0323-F & $\begin{array}{l}\text { AGGGAACAAAAGCTGGGTACGATTTAAGAAGGAGATATACATATGTTTAAA } \\
\text { TATACATATGACATAT }\end{array}$ \\
\hline PAK-0323-R & CAGGAATTCGATATCAAGCTTTACTTGTCGTCGTCGTCCT \\
\hline M13-F & TGTAAAACGACGGCCAGT \\
\hline M13-R & CAGGAAACAGCTATGACC \\
\hline esrB-qPCR-F & CGACCAGCTTGAGAATTTGCC \\
\hline esrB-qPCR-R & GTAGCCTCGTCCGATATGGC \\
\hline gyrB-qPCR-F & GGATAACGCGATTGACGAAGC \\
\hline gyrB-qPCR-R & CTGTACGGAGACGGAGTTGT \\
\hline eseC-qPCR-F & GGGGCCAAAGATGTCCAGAA \\
\hline eseC-qPCR-R & ATCACGATCCTAAGCGCGAG \\
\hline eseB-qPCR-F & CCCGCTTTCTTGAACTTGGC \\
\hline eseB-qPCR-R & ACGCTATTCACCGATCTGGC \\
\hline
\end{tabular}

samples for SDS-PAGE were prepared by washing the cells with $\mathrm{PBS}$ and boiling them together with protein loading buffer at $100{ }^{\circ} \mathrm{C}$ for $10 \mathrm{~min}$. To obtain the extra cellular protein (ECP) samples, cell-free supernatants were concentrated by a centrifugal filter device (Amicon Ultra-15, Millipore) with a $10-\mathrm{kDa}$ molecular weight cut-off. To harvest the cytoplasm (Cyto) and membrane protein (Mem) samples, the bacterial cells were broken by high pressure homogenizer (Union) at $4{ }^{\circ} \mathrm{C}$ for 5 min under the pressure of 700 bar. After centrifugation, the supernatant was collected as Cyto, while the precipitation was collected as Mem.

\section{Adherence and internalization assays}

HeLa cells (ATCC CCL-2) were cultured in 24-well plates at $37{ }^{\circ} \mathrm{C}$ with $5 \%(\mathrm{v} / \mathrm{v}) \mathrm{CO}_{2}$. Bacterial cells were incubated in DMEM at $30^{\circ} \mathrm{C}$ for $12 \mathrm{~h}$ without shaking, then washed twice with PBS and added into HeLa cells at a multiplicity of infection (MOI) of 100:1. The plates were incubated at $35^{\circ} \mathrm{C}$ for $2 \mathrm{~h}$, followed by adding gentamycin for $0.5 \mathrm{~h}$ to kill extracellular bacteria. To quantify the number of bacteria invaded into HeLa cells, $1 \%$ (v/v) Triton X-100 was added to lyse HeLa cells for $0.5 \mathrm{~h}$ and the lysate was washed for three times with PBS. After that, the lysate was diluted ten times and plated on LB agar medium containing Col. After $24 \mathrm{~h}$, bacterial colonies on plates were counted to quantify the adherence and internalization efficiency. Each experiment was performed more than three times.

\section{Virulence analysis of E. piscicida}

Bacterial cells were cultured in DMEM at $30{ }^{\circ} \mathrm{C}$ for $12 \mathrm{~h}$ without shaking. Then the bacteria were washed three times with PBS and diluted to $1 \times 10^{5} \mathrm{CFU} / \mathrm{mL}$. Zebrafish purchased from the commercial fish farm (Yantai, China) were acclimatized to laboratory conditions at $25{ }^{\circ} \mathrm{C}$ for 2 weeks. 
EIB202 bacterial cells with different genotypes were injected into zebrafish intraperitoneally (i.p.) at a dose of $500 \mathrm{CFU} / \mathrm{fish}$. The mortality of the fish was recorded every $12 \mathrm{~h}$ after infection. Five fish of each group were chosen and weighed after $48 \mathrm{~h}$ and $96 \mathrm{~h}$, then the fish were broken using MiniBeadBeater-16 (BioSpec USA). After that, the lysates were diluted 10 times, 100 times, 1000 times, 10,000 times, respectively, and plated on LB agar medium containing Col. After $24 \mathrm{~h}$, bacterial colonies on the plates were counted to quantify. Each experiment was performed more than three times. All animal experiments were approved by the Animal Care and Use Committee of East China, University of Science and Technology.

\section{Bioinformatical and statistical analyses}

Domains of InV were predicted by NCBI tool "Identify Conserved Domains" at https://www.ncbi.nlm.nih.gov/Structure/ cdd/wrpsb.cgi?INPUT_TYPE. Sequence alignment was performed by the program ClustalX.

Statistical analysis was performed using GraphPad Prism version 6.0 Software (San Diego, USA). The Tukey-Kramer test and one-way ANOVA was used to analyze the data. Results were shown as mean $\pm \mathrm{SD}$. $* P<0.05$, $* * P<0.01$, $* * * P<0.001$ were identified as significant differences.

\section{Results}

\section{Screen for T3SS up-stream mediators}

As one of the core secretion systems for E. piscicida to invade host cells, up-stream mediators of T3SS have rarely been explored. Previously we constructed the Himarl transposon insertion mutant library of E. piscicida (Wei et al. 2019), in which each mutant has a random but defined transposon insertion locus. Here we performed autoaggregation assay with this library to screen for up-stream T3SS mediators. The rationale for autoaggregation assay is explained in Fig. 1a. EseB is a T3SS translocon component in E. piscicida which can form filamentous appendages on the surface of E. piscicida and facilitate bacterial adhesion to an abiotic surface (Gao et al. 2015). During screening, autoaggregation assay was performed in 96-well plates and the small amount of medium volume in each well caused some extent of bacterial sedimentation (Fig. 1b, white plaque in the middle, referred as "button"). When T3SS is complete, the secreted EseB mediates the adhesion and clump of all bacterial cells. The weight makes them settle down to the bottom (forming "carpet", fuzzing up the white plaque). However, when T3SS is disrupted, no EseB will be secreted. Therefore, cells will not clump and the bottom will be clear ("button" only). As a result, the transposon insertion mutant of the
ETAE_0323 gene (ETAE_0323::Tn) showed dramatic T3SS defect (Fig. 1b). The autoaggregation phenotype was also confirmed in test tubes. Aggregation look in test tubes was slightly different from that in 96-well plates. Because of the larger medium volume in test tubes, natural sedimentation did not happen. Bacteria settled down to the bottom with a functional T3SS, and left the bottom clear when T3SS was disrupted. As shown in Fig. 1c, ETAE_0323::Tn displayed the same autoaggregation phenotype with the $\Delta e s r B$ strain, with much fewer cells accumulated at the bottom. EsrB is a key regulator of T3/T6SS in E. piscicida which plays a crucial role in E. piscicida pathogenicity (Liu et al. 2017). In addition, we collected and concentrated the extracellular proteins (ECPs) for SDS-PAGE. Compared with WT, $\Delta e s r B$ strain showed defect on the ECPs from both T3SS (EseB, EseC, and EseD) and T6SS (EvpI, EvpP, and EvpC). Interestingly, the transposon mutant ETAE_0323::Tn abolished the secretion through T3SS but had an unaffected T6SS (Fig. 1d, e), suggesting that ETAE_0323 may specifically activate T3SS.

\section{Bioinformatics prediction of ETAE_0323 structure and function}

ETAE_0323 encoded a protein with 2359 amino acid residues, and was annotated as an invasin (Wang et al. 2009). We then renamed it $\mathrm{InV}$ and performed domain prediction using the NCBI online tool. Similar to most invasins and intimins, InV mainly contained three different domains (from $\mathrm{N}$ to $\mathrm{C}$ terminus): lysin motif (97-143 aa), the $\beta$-barrel porin-like outer membrane domain (183-452 aa), and 16 repeated bacterial Ig-like domains (587-2219 aa) (Fig. 2a). To further characterize the feature of $\mathrm{InV}$, we aligned its protein sequence with other invasins/intimins family members. InV shared 43\%, 43\%, 41\%, 42\%, 37\%, and 34\% identity to invasin (Yersinia intermedia), bacterial Ig-like domain protein (Edwardsiella ictaluri), bacterial Ig-like domain protein ( $Y$. pseudotuberculosis), inverse autotransporter $\beta$-barrel domain protein (Arsenophonus nasoniae), inverse autotransporter $\beta$-barrel domain protein (Morganella morganii), invasin (Yersinia ruckeri), respectively (Fig. 2b). Furthermore, $\beta$-barrel region (183-452 aa) was the most conserved domain in InV (Fig. 2b). InV had 16 bacterial Iglike domains, making it bigger than most invasins in other bacteria. Alignments also revealed high homology among these 16 Big_3_5 domains of InV (Fig. 2c).

\section{Relationship between InV and T3SS}

To further identify the relationship between $\operatorname{InV}$ and T3SS, N-terminus and C-terminus deletion mutants $\left(\Delta i n V-N^{\prime}\right.$ and $\left.\Delta i n V-C^{\prime}\right)$ were constructed from wildtype EIB202 strain (full length inV was too big to be 
A

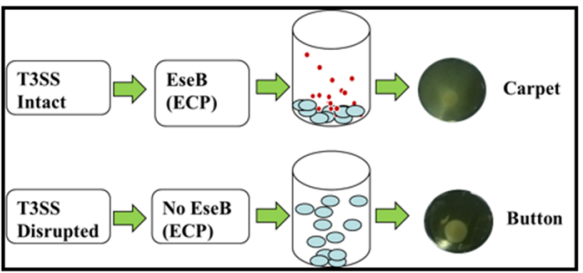

B

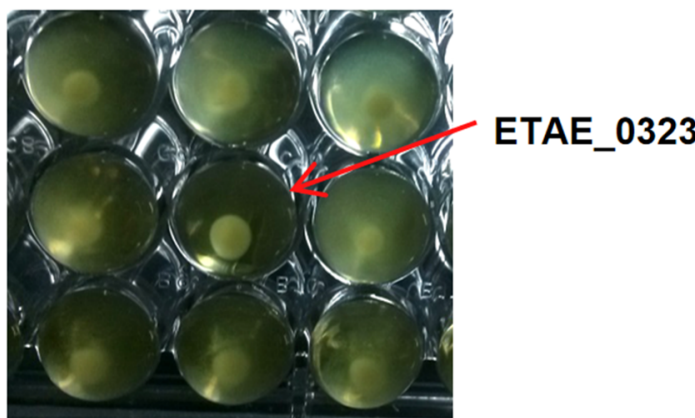

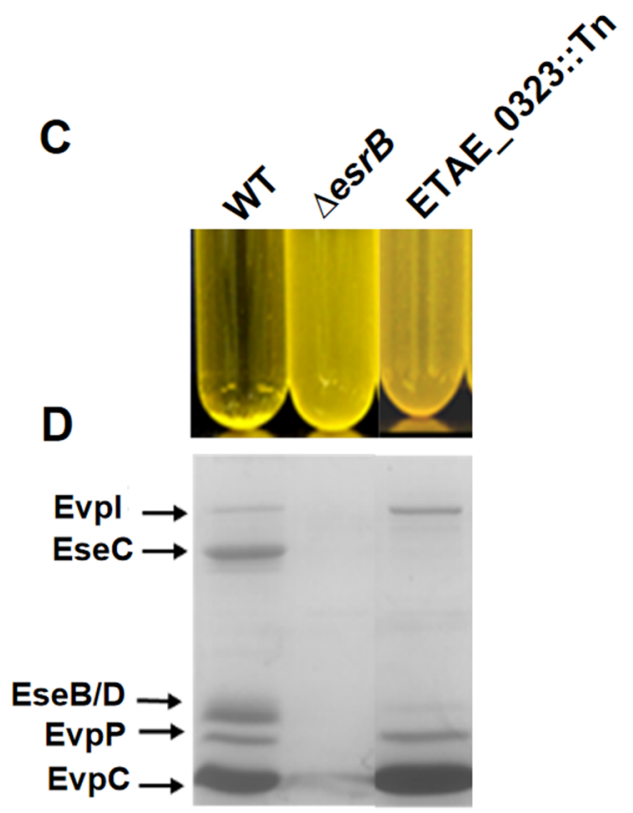

E

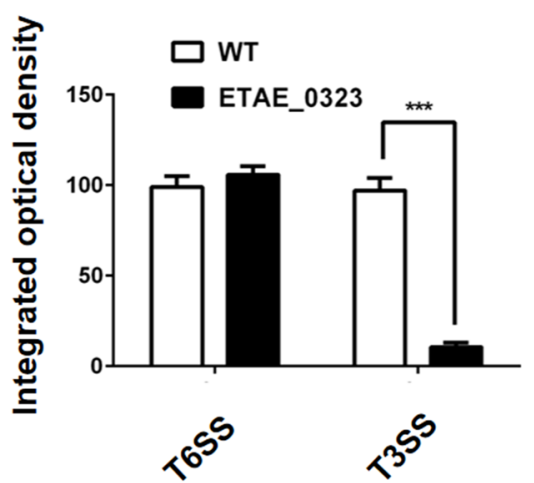

Fig. 1 T3SS mediator screening identified the transposon mutant ETAE_0323::Tn as a potential T3SS deficient strain. a Rationale of the autoaggregation assay to screen for mediators of T3SS via transposon insertion library. b ETAE_0323::Tn from the transposon insertion library showed a desired phenotype. c Autoaggrega-

deleted). Autoaggregation assays were then performed again (Fig. 3a). Unlikely the transposon mutant in $V:: T n$, $\mathrm{N}$-terminus and C-terminus deletion of in $V$ did not affect the phenotype of autoaggregation. To further examine the influence of InV to T3SS, EseB levels were measured in both ECPs and WCPs since it was the major elemental protein of T3SS. As shown by Fig. 3b, EseB secretion rather than expression was disrupted in the transposon mutant in $V:: T n$. However, InV deletion did not affect EseB secretion, as well as expression (lane 4 and lane 5 in Fig. 3b). In addition, qRT-PCR data suggested that the deletion of InV had no influence on T3SS transcription (Fig. 3c). Taken together, these results demonstrate that $\mathrm{InV}$ protein itself affects neither secretion nor expression of T3SS. tion assay of WT, $\Delta e s r B$ and ETAE_0323::Tn strains in test tubes. d Extracellular protein profiles of several typical T3SS (EseB, EseC, EseD) and T6SS (EvpI, EvpP, EvpC) components in WT, $\Delta e s r B$ and ETAE_0323::Tn strains. e Integrated optical density of the protein bands in $\mathbf{d}$

Transposon insertion mutants always result in polar effect and lead to confused phenotypes (Harayama et al. 1983; Dymov et al. 2004). To further elucidate the relationship between InV and T3SS, we expressed Flag tagged InV-N' fragment in EIB202 WT and $\triangle \mathrm{T} 3 \mathrm{SS}$ (eseBCD deletion) strains. InV-N' levels were detected in WCPs, ECPs, cytoplasma proteins (Cyto), and membrane proteins (Mem) through Flag antibody to reveal its localization. According to Fig. 3d (left 4 lanes), in WT cells a majority of InV-N' fragment was located on the membrane with a little bit in cytosol as well, suggesting that $\mathrm{InV}$ was a membrane protein. In addition, no InV-N' $\mathrm{N}^{\prime}$ was secreted out of cells (ECPs). The membrane localization of InV-N' was partially affected by T3SS, however, not significantly when compared with 
A

\begin{tabular}{|c|c|}
\hline inV-N' (1-452aa) & inV-C' (692-2,359aa) \\
\hline
\end{tabular}

LysM (Lysin motif)

$\beta$-barrel ( $\beta$-barrel porin-like outer membrane protein)

Big_3_5 super family (Bacterial Ig-like domain)

B
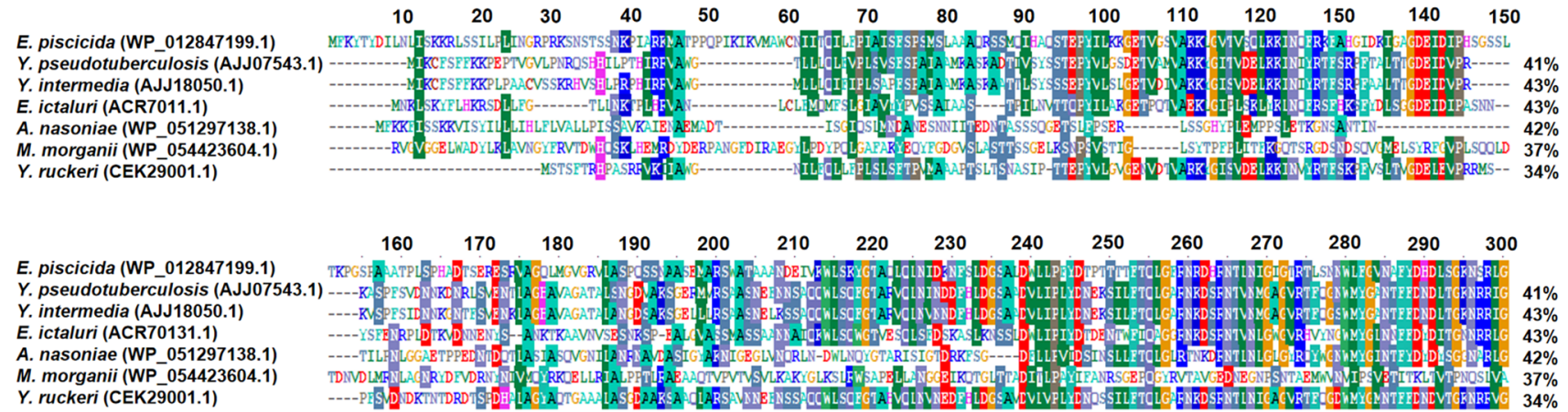

Y. ruckeri (CEK29001.1)

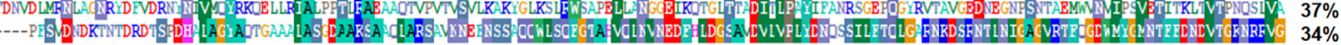

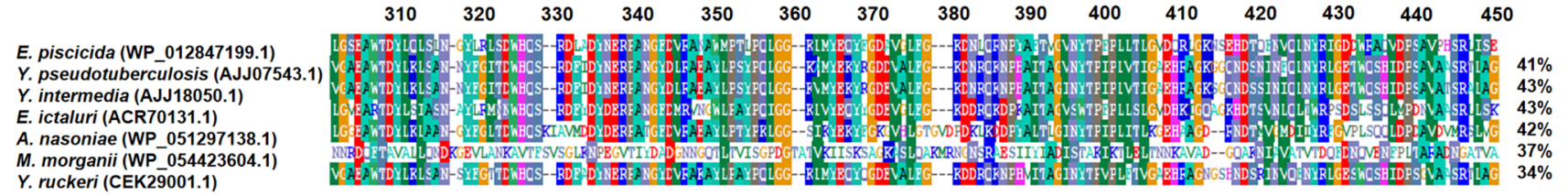

Y. ruckeri (CEK29001.1)

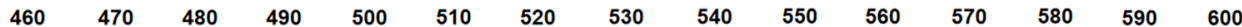

E. piscicida (WP_012847199.1) Y. pseudotuberculosis (AJJ07543.1) Y. intermedia (AJJ18050.1) E. ictaluri (ACR701311)

A. nasoniae (WP 051297138.1)

A.

M. morgani(WP_054423604.1)
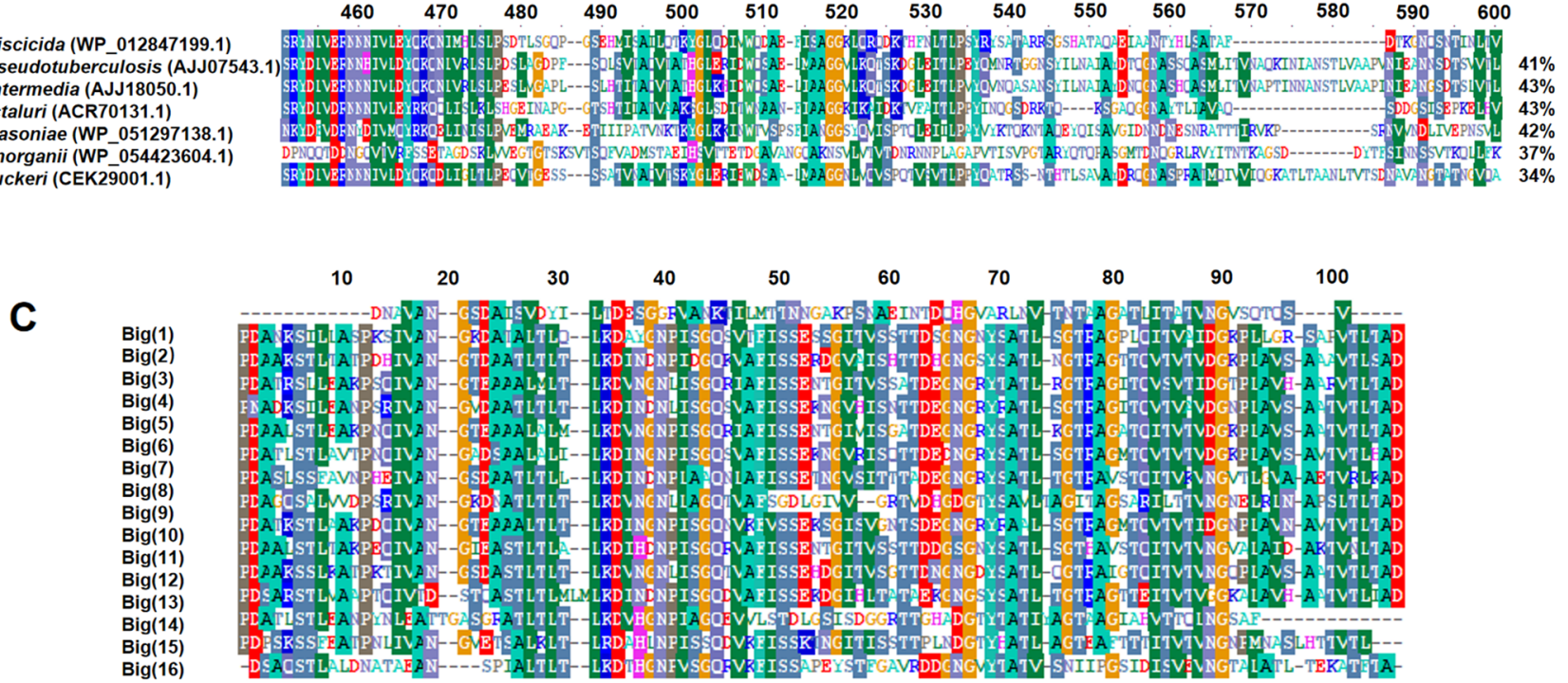

Fig. 2 Sequence analysis of InV in E. piscicida. a Predicted domains of InV predicted by NCBI. b The amino acid sequence at N-terminus of InV was similar to invasins in many other species. Sequences were aligned using BLAST and ClustalX. c Sequence alignment of bacterial Ig-like domains at the C-terminus of InV 
Fig. 3 The relationship between InV and T3SS in E. piscicida. a Autoaggregation assay of WT, $\Delta \operatorname{esr} B$, in $V:: \mathrm{Tn}, \Delta i n V-N^{\prime}$ and $\Delta i n V-C^{\prime}$ strains. $\Delta i n V-N^{\prime}$ and $\Delta i n V-C^{\prime}$ represented in $V$ $\mathrm{N}$-terminal (LysM and $\beta$-barrel domains) and C-terminal (Big_3_5 domains) deleted strains, respectively. $\Delta e s r B$ was used as a T3SS deficient control. All strains were incubated in DMEM at $30{ }^{\circ} \mathrm{C}$ for $24 \mathrm{~h}$ without shaking. b Western blot analysis of the whole cellular proteins (WCPs) and extra cellular proteins (ECPs) in WT, $\Delta e s r B$, in $V:: T n, \Delta i n V-N^{\prime}$ and $\Delta i n V-C^{\prime}$ strains to detect EseB levels. Dnak was used as a control. c qRT-PCR analysis to reveal the transcriptional level changes of esrB, ese $B$ and ese $C$ in $\Delta e s r B, i n V:: T n, \Delta i n V-N^{\prime}$ and $\triangle i n V-C^{\prime}$ strains compared with WT. The experiments were performed in more than three replicates and recorded as mean \pm SD. $* P<0.05$, $* * * P<0.001$. d Anti-flag western blot showing the localization of InV. The N-terminus InV fragment (LysM domain and $\beta$-barrel domain) was fused with a flag tag for western blot detection

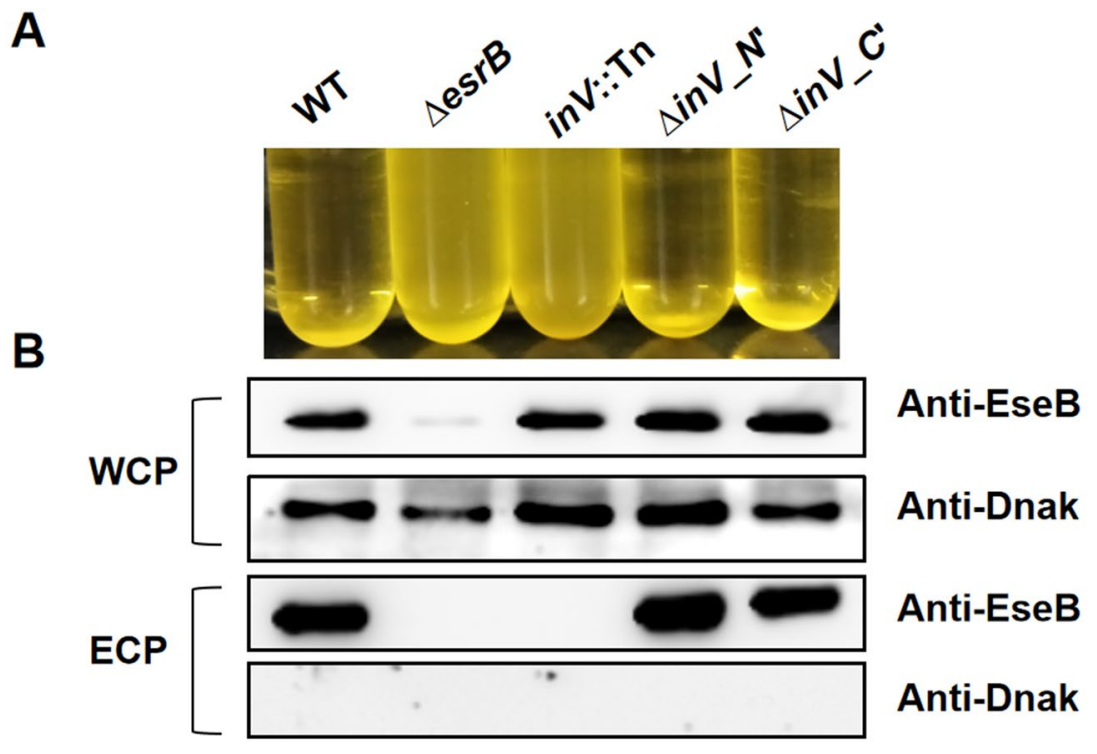

C
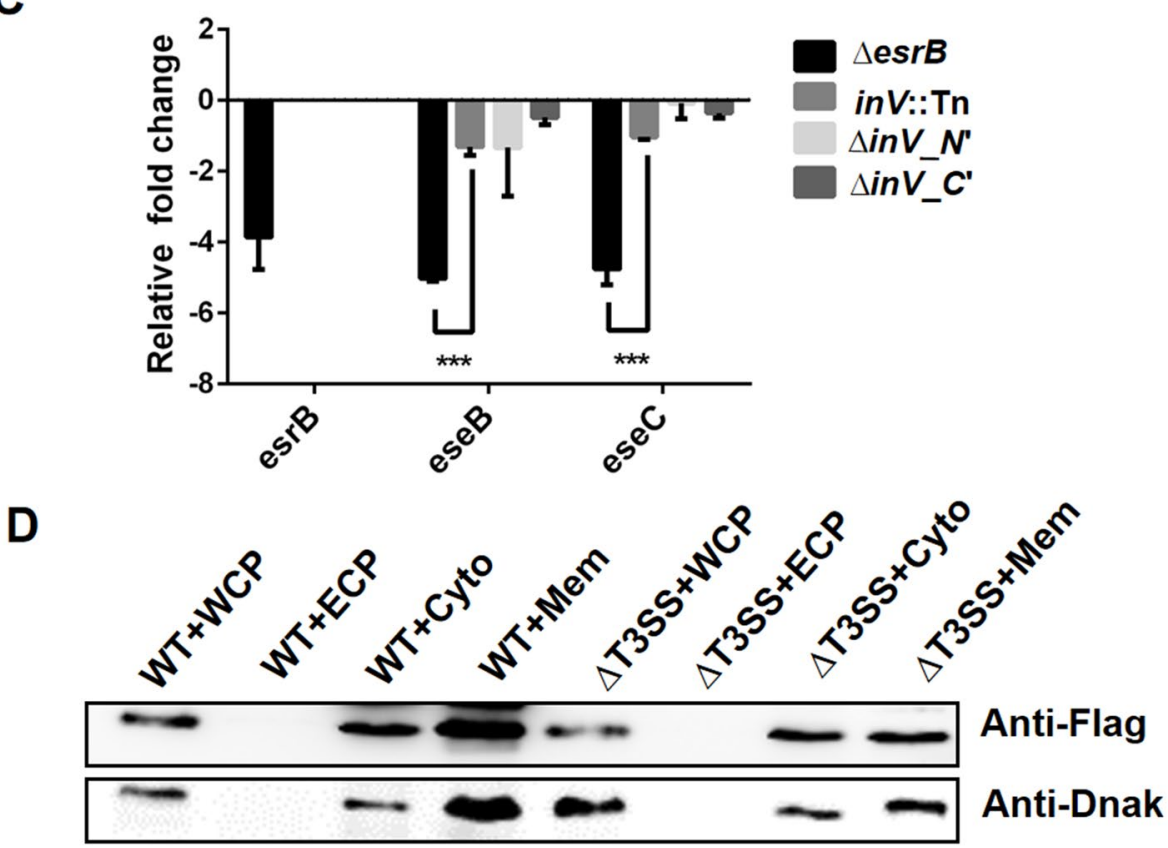

DnaK control (Fig. 3d, right 4 lanes). These data suggest that InV may not have strong relationship with T3SS, and the T3SS depletion phenotypes of in $V:: T n$ may be caused by polar effect.

\section{Contribution of $\ln V$ to pathogen internalization and virulence}

Invasins and intimins were always associated with internalization and infection of the pathogen. To investigate whether InV affects internalization, HeLa cells were infected with the EIB202 strains including WT, $\Delta \mathrm{T} 3 \mathrm{SS}$, in $V:: \mathrm{Tn}, \Delta i n V-N^{\prime}$, $\Delta i n V-C^{\prime}$, and $\Delta i n V-N^{\prime+}$. Internalized bacteria were counted after $2 \mathrm{~h}$ post-infection. As shown by Fig. $4 \mathrm{a}$, the number of internalized bacteria is significantly less in $\Delta \mathrm{T} 3 \mathrm{SS}, \Delta i n V-N^{\prime}$, $\Delta i n V-C^{\prime}$ and in $V::$ Tn groups than WT (7.0-, 5.0-, 4.2-, 4.8fold less than WT, respectively). Additionally, InV-N' fragment complementation restored this internalization defect (Fig. 4a, brown column).

To further evaluate the contribution of $\mathrm{InV}$ in pathogen virulence, the same set of EIB202 strains was injected into zebrafish intraperitoneally (i.p.) at a dose of $500 \mathrm{CFU}$ and the mortality of fish was recorded every $12 \mathrm{~h}$. The mortality of zebrafish infected by WT bacteria was significantly higher than $\Delta \mathrm{T} 3 \mathrm{SS}$ or inV deficient bacteria (Fig. 4b), in $V$ complementation again restored the virulence (Fig. 4b, 
A

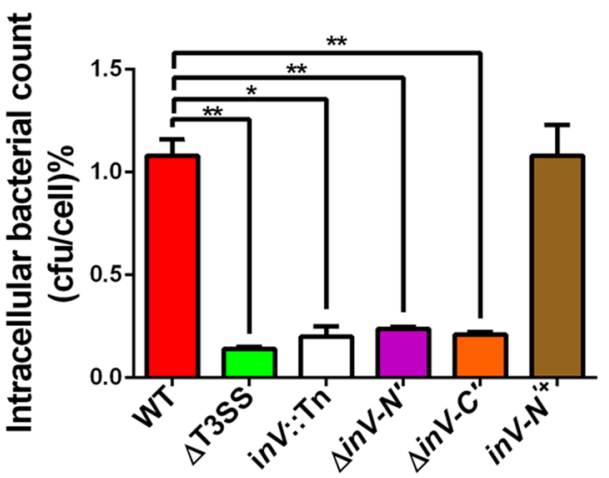

B

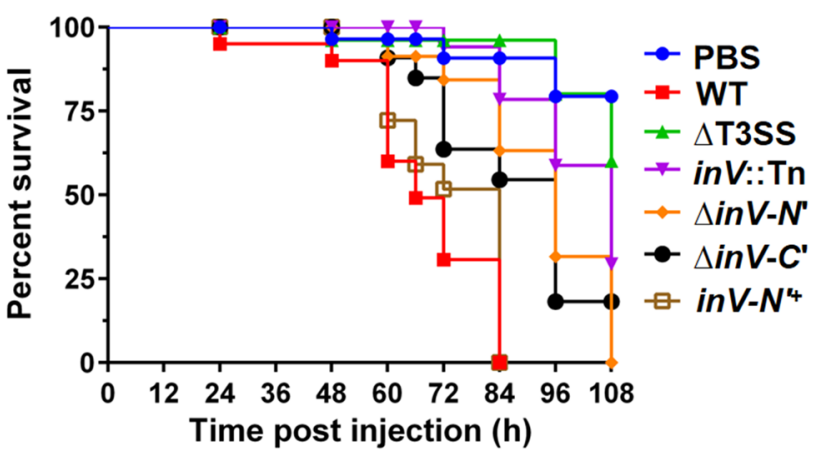

C

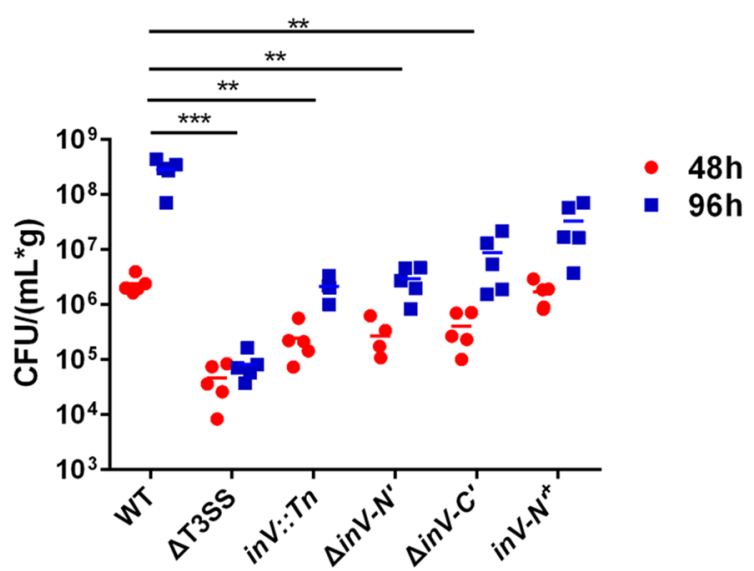

Fig. $4 \mathrm{InV}$ contributes to pathogen internalization and virulence. a WT, $\Delta \mathrm{T} 3 \mathrm{SS}$, in $V:: \mathrm{Tn}, \Delta i n V-N^{\prime}, \Delta i n V-C^{\prime}$ and $i n V-N^{\prime+}$ strains were incubated in DMEM at $30{ }^{\circ} \mathrm{C}$ for $12 \mathrm{~h}$ without shaking. HeLa cells were infected with these strains for $2 \mathrm{~h}$, and the number of bacteria internalized into HeLa cells was determined by plate count. The experiments were performed in more than three replicates and recorded as mean \pm SD. $* P<0.05, * * P<0.01$. b Zebrafish survival curves after PBS or EIB202 strains with various genotypes (WT, $\Delta \mathrm{T} 3 \mathrm{SS}$, in $V:: \mathrm{Tn}, \Delta i n V-N^{\prime}, \Delta i n V-C^{\prime}$ and $\left.i n V-N^{\prime+}\right)$ were injected intraperitoneally (i.p.) at a dose of $500 \mathrm{CFU} /$ fish. Fish mortality was recorded every $12 \mathrm{~h}$. c CFU counts of the zebrafish infection assay in B. Experiments were performed by more than three times and the number was recorded as mean \pm SD. $* * P<0.01, * * * P<0.001$ brown curve). Moreover, CFU counts of zebrafish infected by $\Delta \mathrm{T} 3 \mathrm{SS}$ or in $V$ deficient bacteria after $48 \mathrm{~h}$ and $96 \mathrm{~h}$ were significantly lower than WT (Fig. 4c). Similarly, inV complementation successfully reversed the phenotype (Fig. 4c). Taken together, our results demonstrate that InV was involved in internalization and virulence of E. piscicida.

\section{Discussion}

In this study, InV was screened out as significantly correlated with T3SS based on an E. piscicida transposon insertion mutant library. However, both N-terminus and C-terminus deletion of in $V$ were not able to copy the T3SS deficient phenotype of the transposon insertion mutant. We also examined the InV-N' fragment localization and found that it was not significantly affected by T3SS. Therefore, the T3SS defect phenotype of the transposon insertion mutant was likely caused by the polar effect or frameshift mutation induced by transposon (Dymov et al. 2004). It is also likely that $\mathrm{N}$-terminus and $\mathrm{C}$-terminus have redundant functions with T3SS and the full-length protein needs to be depleted to show the phenotype.

Although In $\mathrm{V}$ deletion did not show strong relationship with T3SS, the EIB202- $\Delta i n V-N^{\prime}$ and EIB202- $\Delta i n V-C^{\prime}$ strains showed critical internalization defect towards HeLa cells and virulence attenuation towards zebrafish. Since both the $\mathrm{N}$-terminus deletion and $\mathrm{C}$-terminus deletion of in $\mathrm{V}$ showed internalization and virulence defect, it is likely that LysM/ $\beta$-barrel (N-terminus) and bacterial Ig-like domains (C-terminus) were essential for the function of InV. As a newly identified invasin, InV may help with E. piscicida vaccine development since its deletion severely affected pathogen virulence.

Acknowledgements This work was supported by grants from National Natural Science Foundation of China (31672696), Ministry of Agriculture of China (CARS-47), Shanghai Pujiang Program (16PJD018), the Science and Technology Commission of Shandong and Shanghai Municipality (2017CXGC0103 and 17391902000).

\section{Compliance with ethical standards}

Conflict of interest The authors declare that they have no conflict of interest.

\section{References}

Batchelor M, Prasannan S, Daniell S, Reece S, Connerton I, Bloomberg G, Dougan G, Frankel G, Matthews S (2000) Structural basis for recognition of the translocated intimin receptor (Tir) by intimin from enteropathogenic Escherichia coli. EMBO J 19:2452-2464 
Buist G, Steen A, Kok J, Kuipers OP (2010) LysM, a widely distributed protein motif for binding to (peptido) glycans. Mol Microbiol 68:838-847

Chauhan N, Wrobel A, Skurnik M, Leo JC (2016) Yersinia adhesins: an arsenal for infection. Proteomics Clin Appl 10:949-963

Dymov SI, Meek DJ, Steven B, Driscoll BT (2004) Insertion of transposon Tn5tac1 in the Sinorhizobium meliloti malate dehydrogenase $(m d h)$ gene results in conditional polar effects on downstream TCA cycle genes. Mol Plant Microbe Interact 17:1318-1327

Fairman JW, Dautin N, Wojtowicz D, Liu W, Noinaj N, Barnard TJ, Udho E, Przytycka TM, Cherezov V, Buchanan SK (2012) Crystal structures of the outer membrane domain of intimin and invasin from enterohemorrhagic E. coli and enteropathogenic $Y$. pseudotuberculosis. Structure 20:1233-1243

Fan E, Chauhan N, Udatha G, Leo J, Linke D (2016) Type V secretion systems in bacteria. Microbiol Spectr 4:1-24

Gao ZP, Nie P, Lu JF, Liu LY, Xiao TY, Liu W, Liu JS, Xie HX (2015) Type III secretion system translocon component EseB forms filaments on and mediates autoaggregation of and biofilm formation by Edwardsiella tarda. Appl Environ Microbiol 81:6078-6087

Gillenius E, Urban CF (2015) The adhesive protein invasin of Yersinia pseudotuberculosis induces neutrophil extracellular traps via $\beta 1$ integrins. Microbes Infect 17:327-336

Green DM (2010) A strategic model for epidemic control in aquaculture. Prev Vet Med 94:119-127

Harayama S, Bollinger J, Iino T, Hazelbauer GL (1983) Characterization of the $\mathrm{mgl}$ operon of Escherichia coli by transposon mutagenesis and molecular cloning. J Bacteriol 153:408-415

Heinz E, Stubenrauch CJ, Grinter R, Croft NP, Purcell AW, Strugnell RA, Dougan G, Lithgow T (2016) Conserved features in the structure, mechanism, and biogenesis of the inverse autotransporter protein family. Genome Biol Evol 8:1690-1705

Hu T, Chen R, Zhang L, Wang Z, Yang D, Zhang Y, Liu X, Liu Q (2019) Balanced role of T3SS and T6SS in contribution to the full virulence of Edwardsiella piscicida. Fish Shellfish Immunol 93:871-878

Isberg RR, van Nhieu GT (1995) The mechanism of phagocytic uptake promoted by invasin-integrin interaction. Trends Cell Biol 5:120-124

Leibiger K, Schweers JM, Schütz M (2019) Biogenesis and function of the autotransporter adhesins YadA, intimin and invasin. Int $\mathbf{J}$ Med Microbiol 309:331-337

Leo JC, Grin I, Linke D (2012) Type V secretion: mechanism(s) of autotransport through the bacterial outer membrane. Philos Trans R Soc Lond B Biol Sci 367:1088-1101

Leo JC, Oberhettinger P, Schütz M, Linke D (2015) The inverse autotransporter family: intimin, invasin and related proteins. Int J Med Microbiol 305:276-282

Leotta GA, Piñeyro P, Serena S, Vigo GB (2009) Prevalence of Edwardsiella tarda, in antarctic wildlife. Polar Biol 32:809-812

Leung KY, Siame BA, Tenkink BJ, Noort RJ, Mok Y (2012) Edwardsiella tarda - virulence mechanisms of an emerging gastroenteritis pathogen. Microbes Infect 14:26-34

Leung KY, Wang Q, Yang Z, Siame BA (2019) Edwardsiella piscicida: a versatile emerging pathogen of fish. Virulence 10(1):555-567

Liu Y, Zhao L, Yan M, Yin K, Zhou X, Leung KY, Liu Q, Zhang Y, Wang Q (2017) Transcriptomic dissection of the horizontally acquired response regulator EsrB reveals its global regulatory roles in the physiological adaptation and activation of T3SS and the cognate effector repertoire in Edwardsiella piscicida during infection toward turbot. Virulence 8:1355-1377

Liu L, Fang H, Ding Y, Zheng Y, Cai L, Zheng S, Zhang Y (2018) Transcriptional regulation between the two global regulators RovA and CRP in Yersinia pestis biovar microtus. Curr Microbiol 75:1634-1641

Martens E, Demain AL (2017) An overview of the industrial aspects of antibiotic discovery, chapter 7. Microbial resources, pp 149-168
Oberhettinger P, Schutz M, Leo JC, Heinz N, Berger J, Autenrieth IB, Linke D (2012) Intimin and invasin export their C-terminus to the bacterial cell surface using an inverse mechanism compared to classical autotransport. PLoS ONE 7:e47069

Okuda J, Kiriyama M, Suzaki E, Kataoka K, Nishibuchi M, Nakai T (2009) Characterization of proteins secreted from a type III secretion system of Edwardsiella tarda and their roles in macrophage infection. Dis Aquat Organ 84:115-121

Park SB, Aoki T, Jung TS (2012) Pathogenesis of and strategies for preventing Edwardsiella tarda infection in fish. Vet Res 43:67

Picardeau M (2010) Transposition of fly mariner elements into bacteria as a genetic tool for mutagenesis. Genetica 138:551-558

Sadana P, Geyer R, Pezoldt J, Helmsing S, Huehn J, Hust M, Dersch P, Scrima A (2018) The invasin D protein from Yersinia pseudotuberculosis selectively binds the Fab region of host antibodies and affects colonization of the intestine. J Biol Chem 293:8672-8690

Srinivasa Rao PS, Lim TM, Leung KY (2003) Functional genomics approach to the identification of virulence genes involved in Edwardsiella tarda pathogenesis. Infect Immun 71:1343-1351

Srinivasa Rao PS, Yamada Y, Tan YP, Leung KY (2004) Use of proteomics to identify novel virulence determinants that are required for Edwardsiella tarda pathogenesis. Mol Microbiol 53:573-586

Tan YP, Lin Q, Wang XH, Joshi S, Hew CL, Leung KY (2002) Comparative proteomic analysis of extracellular proteins of Edwardsiella tarda. Infect Immun 70:6475-6480

Tsai JC, Yen MR, Castillo R, Leyton DL, Henderson IR, Saier MJ (2010) The bacterial intimins and invasins: a large and novel family of secreted proteins. PLoS ONE 5:e14403

Van Ulsen P, Zinner KM., Jong, WS, Luirink J (2018) On display: autotransporter secretion and application. FEMS Microbiol Lett 365(18)

Wang Q, Yang M, Xiao J, Wu H, Wang X, Lv Y, Xu L, Zheng H, Wang S, Zhao G, Liu Q, Zhang Y (2009) Genome sequence of the versatile fish pathogen Edwardsiella tarda provides insights into its adaptation to broad host ranges and intracellular niches. PLoS ONE 4:e7646

Wei L, Wu Y, Qiao H, Xu W, Zhang Y, Liu X (2018) YebC controls virulence by activating T3SS gene expression in the pathogen Edwardsiella piscicida. FEMS Microbiol Lett 365:14

Wei L, Qiao H, Sit B, Yin K, Yang G, Ma R, Ma J, Yang C, Yao J, Ma Y, Xiao J, Liu X, Zhang Y, Waldor MK, Wang Q (2019) A bacterial pathogen senses host mannose to coordinate virulence. iScience 20:310-323

Xiao J, Wang Q, Liu Q, Wang X, Liu H, Zhang Y (2008) Isolation and identification of fish pathogen Edwardsiella tarda from mariculture in China. Aquac Res 40:13-17

Yang M, Lv Y, Xiao J, Wu H, Zheng H, Liu Q, Zhang Y, Wang Q (2012) Edwardsiella comparative phylogenomics reveal the new intra/inter-species taxonomic relationships, virulence evolution and niche adaptation mechanisms. PLoS ONE 7:e36987

Yang W, Wang L, Zhang L, Qu J, Wang Q, Zhang Y (2015) An invasive and low virulent Edwardsiella tada EsrB mutant promising as live attenuated vaccine in aquaculture. Appl Microbiol Biotechnol 99:1765-1777

Zheng J, Tung SL, Leung KY (2005) Regulation of a type III and a putative secretion system in Edwardsiella tarda by EsrC is under the control of a two-component system, EsrA-EsrB. Infect Immun 73:4127-4137

Publisher's Note Springer Nature remains neutral with regard to jurisdictional claims in published maps and institutional affiliations. 